LBL-36061

CBP Note-101

A Proposal for the Generation of Ultra-Short X-Ray Pulses*

\author{
Alexander A. Zholents \\ Center for Beam Physics \\ Accelerator and Fusion Research Division \\ Lawrence Berkeley Laboratory \\ University of California \\ Berkeley, CA 94720 \\ and
}

Max S. Zolotorev

Stanford Linear Accelerator Center

Stanford University

Stanford, CA 94309

Presented at the 16th Free Electron Laser International Conference Stanford University, August 21-26, 1994

* This work was supported by the Director, Office of Energy Research, Office of High Energy and Nuclear Physics, High Energy Physics Division, of the U.S. Department of Energy under Contract No. DE-AC03-76SF00098 and DE-AC03-76SF00515. 


\title{
A proposal for the generation of ultra-short x-ray pulses
}

\author{
Alexander A. Zholents ${ }^{1}$, and Max S. Zolotorev ${ }^{2}$ \\ 1) Center for Beam Physics, Lawrence Berkeley Laboratory, University of California, Berkeley, CA 94720 \\ 2) Stanford Linear Accelerator Center, Stanford University, Stanford, CA 94309

\begin{abstract}
In this paper it is shown that optical stochastic cooling in a $150 \mathrm{MeV}$ electron storage ring will allow production of a beam with longitudinal emittance $4.5 \times 10^{-6} \mathrm{MeV} \cdot \mathrm{m}$. Such a small emittance accompanied with a bunch compression technique based upon the transformation in the longitudinal phase space will allow achieving a bunch length $30 \mu \mathrm{m}$. This bunch could then be used for the generation of ultra-short $\mathrm{x}$-ray pulses by the Compton scattering of laser photons.
\end{abstract}

\section{Introduction}

In this paper we briefly summarize a feasibility study of a $x$-ray source capable of producing x-ray pulses with a duration $\sigma_{\tau}=100 \mathrm{fs}$, repetition rate. $714 \mathrm{MHz}$ and average flux $3 \times 10^{11} \mathrm{x}$-rays/sec [1]. X-rays are generated by Compton scattering photons from a laser on electrons circulating in the storage ring. The ultra-short duration of the $x$-ray pulses is due to the short length of the electron bunches in the laser/e-beam interaction region (IR). A compression and decompression of the electron bunches before and after the IR helps to form short bunches in the IR while keeping them long in other sections of 


\section{DISCLAIMER}

This report was prepared as an account of work sponsored by an agency of the United States Government. Neither the United States Government nor any agency thereof, nor any of their employees, make any warranty, express or implied, or assumes any legal liability or responsibility for the accuracy, completeness, or usefulness of any information, apparatus, product, or process disclosed, or represents that its use would not infringe privately owned rights. Reference herein to any specific commercial product, process, or service by trade name, trademark, manufacturer, or otherwise does not necessarily constitute or imply its endorsement, recommendation, or favoring by the United States Government or any agency thereof. The views and opinions of authors expressed herein do not necessarily state or reflect those of the United States Government or any agency thereof. 


\section{DISCLAIMER}

Portions of this document may be illegible in electronic image products. Images are produced from the best available original document. 
the ring. The novel optical stochastic cooling technique $[2,3]$ is implemented in this project in order to ensure a small longitudinal beam emittance. This technique is also used for a weak excitation of transverse emittances in order to decrease heating of the longitudinal emittance by intrabeam scattering. The high repetition rate of the $\mathrm{x}$-ray pulses and the high average flux of $x$-rays are due to a) many bunches in the storage ring, b) the usage of a powerful $\mathrm{cw} \mathrm{CO}_{2}$ laser, and c) electron-photon interactions inside the optical resonator.

\section{Storage ring}

Figure 1 shows a layout of the storage ring. Its essential parts are a cooling insertion, a system of bunch compression and decompression, and an interaction region.

The design of the cooling insertion is based on the transit time method of the optical stochastic cooling. Although, the working principle of this method is fully described in [3], for the sake of the self sufficiency of the paper we will repeat here some basic details.

Optical stochastic cooling is essentially a stochastic cooling method and it obeys the same cooling principles as the microwave stochastic cooling. All modifications are associated with the transition to the optical frequency regime. Undulators serve as the pickup and kicker and the amplifier is an optical amplifier, typically dye or Ti:Al2 $\mathrm{O}_{3}$. The cooling insertion by itself includes two undulators, an optical amplifier and a bypass (see the corresponding part of the ring in the Figure 1). Moving along the insertion, the particle radiates an electromagnetic (EM) wave in the first undulator. This wave goes to the optical amplifier, while the particle follows the bypass trajectory and meets its amplified radiation in the second undulator. A subsequent interaction between the particle and the EM wave from its own amplified radiation results in a change of the particle energy. The amount of the energy change depends on the amplitude and phase of the EM 
wave at the time when the particle begins to absorb energy in the undulator. In our scheme we hold the amplitude fixed and rely on the phase variation.

The variation of the above mentioned phase from particle to particle is due only to the particle transit time in the bypass, since EM waves radiated by different particles propagate from the first undulator to the second undulator identically. Therefore, in order to have the energy change proportional to, for example, the particle momentum deviation one needs to do the following: $i$ ) adjust the propagation time of the EM wave and the traveling time of a particle with a zero momentum deviation so that this particle enters the second undulator at zero electric field and thus does not undergo any energy change; ii) design the bypass optics such that particles with different momenta follow trajectories with different path lengths, so they enter the second undulator with phase shifts (relative to the phase with zero electric field) proportional to their momentum deviation.

A similar approach is applicable to betatron motion. What is required in this case is to link the particle traveling time in the bypass with some quantitative characteristic of the betatron motion (say, the betatron coordinate or angle or a linear combination of them) at the beginning of the bypass and to install undulators in a region with a non-zero dispersion function.

We have so far considered the interaction of the arbitrary test particle with the EM wave of its own radiation - the so-called coherent kick that the particle receives in the second undulator. However, each particle also interacts with the EM waves emitted by other particles that are within a distance defined by the bandwidth, $\Gamma$, of the amplifier. These interactions constitute the incoherent kick received by the particle. Choosing the amplification factor of the amplifier one can maintain a proper balance between coherent damping and incoherent heating leading to the minimum damping time. At the optimum and when the amplification factor is not limited by the amplifier power, the damping time of synchrotron oscillations, $\tau_{s}$, is given by [2]:

$\tau_{s}=\frac{N}{\Gamma} \frac{\lambda}{F \ell_{b}} T$ 
where $N$ is the number of particles in a bunch, $\ell_{b}$ is the bunch length, $\lambda$ is the wavelength of the undulator radiation, $T$ is the revolution period (we assume only one cooling insertion in the ring), and $F$ is the ratio of the beam transverse area in the undulator to the difraction limited area of the light. Notice, that if $F>1$, then second order chromatic and geometric aberrations need to be corrected with sextupoles in order to fully use this factor for reduction of the damping time.

In the current design we have $N=1 \times 10^{9}, \ell_{b}=2.5 \mathrm{~cm}, \lambda=0.8 \mu \mathrm{m}, \Gamma=10 \%, F=50$, and $T=170 \mathrm{~ns}$. Thus, optical stochastic cooling could provide damping time $\tau_{s} \approx 1 \mathrm{~ms}$. It will require $\mathrm{Ti}^{\mathrm{A}} \mathrm{Al}_{2} \mathrm{O}_{3}$ amplifier with $0.3 \mathrm{~W}$ of the average output power.

It follows from eq (1), that the damping time depends inversely from the bunch length. Therefore, a long bunch in the cooling insertion is preferable. At the same time, we need a short bunch in the interaction region (IR) for the generation of short x-ray pulses. The only way to have long and short bunches in one ring is by having bunch compression before the IR and bunch decompression after the IR. Thus, longitudinal phase space transformations similar to the conventional bunching and debunching (which are typical for transfer lines) must be implemented in the ring. Basically, this means that the ring must possess a large momentum compaction factor. This is done in our design by adding $\alpha$-rotators to the lattice. Additionally, two high voltage RF cavities, located before and after the cooling insertion, serve as the energy demodulator and modulator. As a result, this system is capable of creating a significant variation of the longitudinal beta-function along the ring with a ratio of the longitudinal beta-function in the cooling system, $\beta_{1}$, to the longitudinal beta-function in the IR, $\beta_{2}$ given by:

$\beta_{1} / \beta_{2} \cong\left[\pi\left(0.5-Q_{s}\right)\right]^{-2}$

where $Q_{s}$ is the synchrotron frequency.

We have chosen $Q_{s}$ to be close to the half integer resonance with detuning $\Delta Q_{s}<0.001$ in order to explore the beta-beat enhancement near resonance. Long term stability of the magnetic field and accelerating voltage on the level of $10^{-3}$ seems adequate to maintain 
this condition, although, the resonance harmonics of the magnetic field ripples in the bending magnets and the accelerating voltage ripples in the cavities should be controlled with a better precision of $10^{-4}$ to $10^{-5}$.

Due to a small beam energy spread of $3 \times 10^{-6}$ in the cooling insertion, the strongest excitation of longitudinal emittance comes from intrabeam scattering. Therefore, we decided to operate in a fully coupled regime with the relatively large horizontal and vertical emittances of $1 \times 10^{-6} \mathrm{~m} \cdot \mathrm{rad}$. First, this helps to reduce the growth rates of beam emittances from intrabeam scattering. Second, it helps to decrease the stochastic cooling damping time. For a current variant of the ring lattice and for the beam parameters, listed in Table 1, the growth rate of the longitudinal emittance is estimated to be $750 \mathrm{~s}^{-1}$.

Radiation damping in the ring is only $0.7 \mathrm{sec}$. Although it is slow, it is sufficient to balance transverse emittance excitation associated with the quantum fluctuations of synchrotron radiation and intrabeam scattering.

Table 1 contains a list of the main storage ring parameters. Table 2 contains parameters of the optical amplifier.

\section{X-ray generation}

Electrons interact with laser photons inside an optical resonator, as it shown schematically in Figure 2. The resonator is formed by two confocal mirrors. One mirror is semi-transparent. An external $\mathrm{cw} \mathrm{CO}_{2}$ laser is used for a power buildup in the resonator up to $300 \mathrm{~kW}$. The distance between mirrors and their diameter are optimized in order not to exceed $10 \mathrm{~kW} / \mathrm{cm}^{2}$ of incident power density on the mirror surfaces. The transverse sizes of the electron and photon beams are matched at the waist point. In this case the number of x-rays, $N_{\gamma}$, created in a single pass through the resonator by an electron bunch of $N_{b}$ electrons is given by: 
$N_{\gamma}=\frac{2 \pi}{3} \alpha r_{e} \frac{P}{m c^{3}} N_{b} \approx 1.6 \cdot 10^{-9} P(k W) N_{b}$

where $\alpha$ is the fine structure constant, $r_{e}$ is the classical electron radius, $m$ is the electron mass, $c$ is speed of light, and $P$ is the power of the light beam inside the resonator.

Table 3 contains laser characteristics and parameters of the generated $X$-rays.

\section{Discussion}

An average flux of $\mathrm{x}$-rays as high as $1 \times 10^{12} \mathrm{x}$-rays/sec could potentially be achieved with the scheme described in this paper and $1 \mathrm{MW}$ power in the optical resonator. This would require a new optical resonator with larger distance between mirrors and larger mirror diameters and a more powerful $\mathrm{cw} \mathrm{CO}_{2}$ laser for a power buildup in the resonator. It seems also possible that $x$-ray pulses shorter than described in Table 3 could be generated at the expense of the average flux of $x$-rays.

A unique feature of the proposal discussed in this paper is an extremely small bunch length in the IR of the storage ring. Accordingly, the bunch is also very short in the bending magnets adjacent to the IR. Therefore, the synchrotron radiation from these magnets that is radiated in the far infrared will be substantially coherent. One can explore this feature for an upgrade of the current scheme into a source of a high power far infrared radiation. 


\section{Acknowledgments}

We acknowledge useful discussions with S. Chattopadhyay, K. J. Kim and W. Turner. We express our gratitude to M. Zisman for an extensive introduction to the computer code ZAP [4], which we used for calculations of the intrabeam scattering. This work was supported by Department of Energy contracts DE-AC03-76SF00098 (LBL) and DEAC03-76SF00515 (SLAC).

\section{References}

[1] A. Zholents, M. Zolotorev, CBP Tech Note-038, LBL, (1993)

[2] A. Mikhailichenko, M. Zolotorev, Phys.Rev.Lett. 71, 4146 (1993)

[3] M. Zolotorev, A. Zholents, SLAC-PUB-6476, LBL 35469, (1994) to be published in Phys. Rev. E

[4] M. Zisman, et. al., "ZAP user's manual", LBL-21270, (1986). 
Table 1. Main parameters of the storage ring

\begin{tabular}{|c|c|c|}
\hline Energy & 150 & $\mathrm{MeV}$ \\
\hline Circumference & 50.4 & $\mathrm{~m}$ \\
\hline \multicolumn{3}{|l|}{ Tunes: } \\
\hline horizontal, Qx & 4.8 & \\
\hline vertical, Qy & 3.4 & \\
\hline synchrotron, Qs & 0.499 & \\
\hline Momentum compaction factor & 0.58 & \\
\hline RF frequency & 714 & $\mathrm{MHz}$ \\
\hline Accelerating voltage & 1.3 & MV \\
\hline Number of cavities & 2 & \\
\hline \multicolumn{3}{|l|}{ Emittances: } \\
\hline horizontal & \multicolumn{2}{|c|}{$1 \times 10^{-6} \mathrm{~m} \cdot \mathrm{rad}$} \\
\hline vertical & \multicolumn{2}{|c|}{$1 \times 10^{-6} \mathrm{~m} \cdot \mathrm{rad}$} \\
\hline longitudinal, $\sigma_{\mathrm{E}} \cdot \sigma_{\mathrm{Z}}$ & \multicolumn{2}{|c|}{$4.5 \times 10^{-6} \mathrm{MeV} \cdot \mathrm{m}$} \\
\hline \multicolumn{3}{|l|}{ Damping times: } \\
\hline transverse & 700 & $\mathrm{~ms}$ \\
\hline longitudinal & 1 & $\mathrm{~ms}$ \\
\hline Intrabeam growth rate & 750 & $\sec ^{-1}$ \\
\hline Particles per bunch & \multicolumn{2}{|c|}{$1 \times 10^{9}$} \\
\hline Number of bunches & \multicolumn{2}{|l|}{90} \\
\hline Empty gap & 12.6 & $\mathrm{~m}$ \\
\hline Average beam current & 80 & $\mathrm{~mA}$ \\
\hline \multicolumn{3}{|c|}{ Beam parameters in the interaction region: } \\
\hline bunch length, $\sigma_{\mathrm{Z}} / \mathrm{c}$ & 100 & fs \\
\hline
\end{tabular}




$\begin{array}{lll}\text { energy spread, } \sigma_{\mathrm{E}} / \mathrm{E}_{0} & 9 \cdot 10^{-4} & \\ \text { horizontal beam size, } \sigma_{\mathrm{X}} & 0.6 & \mathrm{~mm} \\ \text { vertical beam size, } \sigma_{\mathrm{y}} & 0.6 & \mathrm{~mm}\end{array}$

Beam parameters in the cooling insertion:

bunch length, $\sigma_{\mathbf{Z}}$

energy spread, $\sigma_{E} / E_{0}$

Compression factor

\section{(1)}


light power inside the resonator

$300 \mathrm{~kW}$

waist size, $\sigma_{\mathbf{r}}$

$0.6 \mathrm{~mm}$

$\mathrm{X}$-ray beam:

wavelength in the forward direction

$0.3 \AA$

macropulse repetition rate

$5.95 \mathrm{MHz}$

macropulse duration

126 ns

micropulse repetition rate

$714 \mathrm{MHz}$

micropulse duration, $\sigma_{\tau}$

100 fs

average flux of $\mathrm{x}$-rays

$3 \times 10^{11} \mathrm{sec}^{-1}$.

spectral width, $\delta \omega / \omega$

$\sim 1$ 


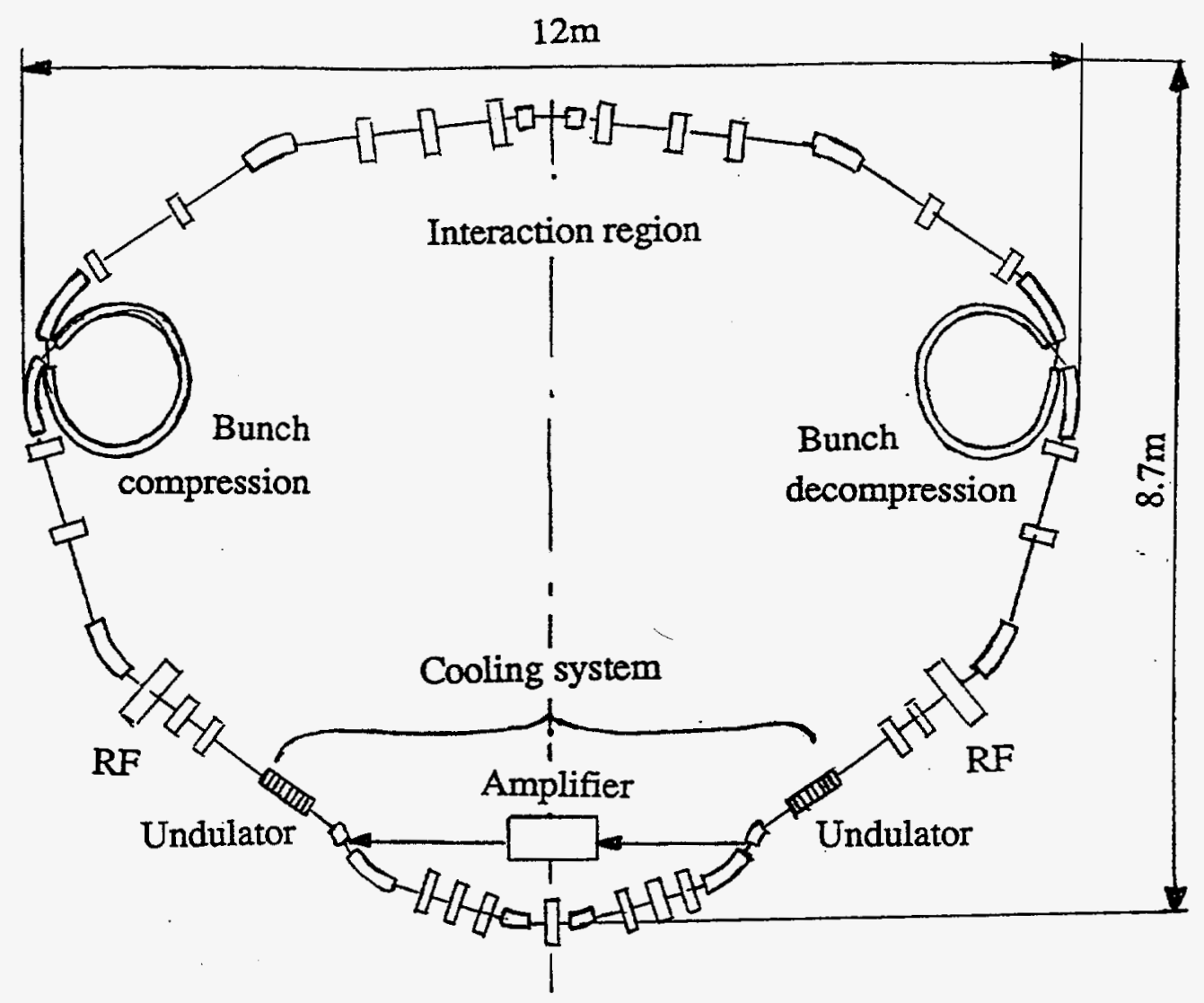

Fig.1 The layout of the storage ring

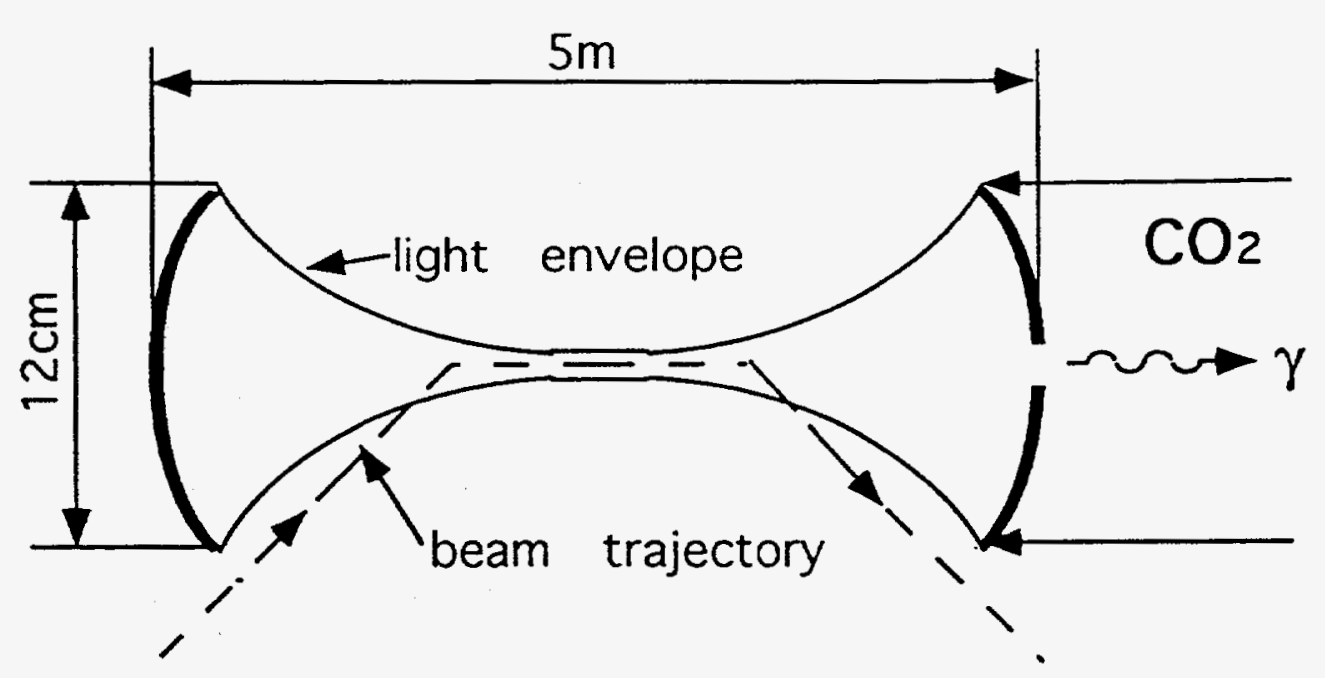

Fig.2 A schematic of electron-photon interaction inside the optical resonator. 